\title{
Gastrointestinal Luminal Stenting: The Early US Experience with the Duodenal HANAROSTENT
}

\author{
Brian M. Funga, ${ }^{a}$ Brian E. Kadera ${ }^{b, c}$ James H. Tabibian ${ }^{b, d}$ \\ ${ }^{a}$ Department of Medicine, Olive View-UCLA Medical Center, Sylmar, CA, USA; ${ }^{b}$ David Geffen School of Medicine at \\ UCLA, Los Angeles, CA, USA; 'Department of Surgery, Olive View-UCLA Medical Center, Sylmar, CA, USA; ${ }^{\mathrm{d} D i v i s i o n}$ of \\ Gastroenterology, Olive View-UCLA Medical Center, Sylmar, CA, USA
}

\section{Keywords}

Gastric outlet obstruction - Gastroduodenal obstruction .

Self-expandable metal stents · Malignant obstruction

\begin{abstract}
Self-expandable metal stents (SEMSs) are frequently utilized for palliation of malignant gastric and/or duodenal outlet obstruction (GDOO). Re-establishing luminal patency with accurate SEMS positioning while limiting migration and adjacent tissue injury is an important technical consideration and aim. The duodenal HANAROSTENT ${ }^{\circ}$ was introduced in the USA in 2019 and developed with these challenges in mind. As the first center in the USA to deploy the duodenal HANAROSTENT $^{\circ}$ in clinical practice, we herein examine our early experience with its use. Specifically, we describe 7 consecutive cases of malignant GDOO in which a duodenal HANAROSTENT ${ }^{\circ}$ was placed for on-label use, defined as palliative treatment of malignant gastric and/or duodenal obstruction. All stents were $22 \mathrm{~mm}$ in diameter, with 5 being 90 $\mathrm{mm}$ and 2 being $120 \mathrm{~mm}$ in length. Technical and clinical success with duodenal HANAROSTENT ${ }^{\circ}$ placement were achieved in all 7 cases (100\%). In no case was stent adjustment required post-deployment. There were no stent-relat-
\end{abstract}

karger@karger.com www.karger.com/gat

Karger $\stackrel{\text { ' }}{5}$

GOPEN ACCESS
(C) 2020 The Author(s)

Published by S. Karger AG, Basel

This is an Open Access article licensed under the Creative Commons Attribution-NonCommercial-4.0 International License (CC BY-NC) (http://www.karger.com/Services/OpenAccessLicense), applicable to the online version of the article only. Usage and distribution for commercial purposes requires written permission. ed adverse events, and no subsequent endoscopic procedures were necessary in any of the patients during a mean follow-up of 5 months (range 1-12 months). In summary, the duodenal HANAROSTENT ${ }^{\circ}$ appears to perform well and be a promising alternative to other available duodenal SEMSs. As experience in the USA with this newly introduced duodenal SEMS grows, multicenter prospective data should be collected to better establish its relative safety and efficacy.

(C) 2020 The Author(s)

Published by S. Karger AG, Basel

\section{Introduction}

Malignant gastroduodenal outlet obstruction (GDOO) refers to the inability of the stomach or duodenal contents to empty as a consequence of obstructing advanced upper gastrointestinal (GI) malignancy or metastatic disease to this anatomical region. Patients with GDOO typically present with nausea, vomiting, unintentional weight loss, and malnutrition [1]. Historically, palliative gastrojejunostomy with or without gastric resection has been performed for cases of GDOO; however, given the often poor nutritional status and prognosis of these patients, surgery 
is often not possible or desirable due to the relatively high perioperative morbidity. Over the years, self-expandable metal stents (SEMSs) have been developed as an alternative to surgery and are now commonly used for the palliation of malignant GDOO, leading to a quicker resumption of oral intake, less morbidity, and reduced inpatient hospital stay [2].

Accurate stent positioning, establishing and maintaining luminal patency, and limiting stent migration and adjacent tissue injury are important considerations in the selection and placement of an SEMS. Data on existing SEMSs suggest that stent design can impact clinical success [3-6]. In 2019, the HANAROSTENT ${ }^{\circledR}$ LowAx $^{\mathrm{TM}}$ Duodenum (Olympus Corporation of the Americas, Center Valley, PA, USA) was approved by the US Food and Drug Administration (FDA) for palliative treatment of pyloric or duodenal obstructions by malignant neoplasms. It was developed to address the challenges associated with duodenal SEMS placement; the unique hook-cross braided nitinol design allows for greater flexibility to improve anatomic conformity, and the incorporation of 12 radiopaque markers at both ends and the middle of the stent aids fluoroscopic visualization and, thus, precise placement (Fig. 1). As the first center in the USA to deploy the HANARO$\mathrm{STENT}^{\circledR}$, we examined our experience and outcomes with its use.

\section{Case Series}

\section{Identification of Patients}

Using a prospectively maintained endoscopic stenting database, we identified all patients who underwent palliative duodenal stent placement with a duodenal HANAROSTENT ${ }^{\circledR}$ between January 2019 and December 2019 at Olive View-UCLA Medical Center, a 377-bed tertiary referral center. GDOO confirmation on computed tomography (CT) of the abdomen and/or upper GI series was required. In all cases, underlying malignancy was confirmed prior to SEMS placement, and the decision to place a palliative SEMS was based on multidisciplinary discussion.

\section{Technical Approach to Stenting}

Each patient had one duodenal HANAROSTENT ${ }^{\circledR}$ deployed. All stents were $22 \mathrm{~mm}$ in diameter by $90 \mathrm{~mm}$ (5 cases) or $120 \mathrm{~mm}$ ( 2 cases) long. All procedures were performed with Olympus therapeutic gastroscopes and with fluoroscopy. The length and anatomical course of stenosis (i.e., obstructed segment) were estimated by pre-procedure imaging. During endoscopy, the length and other features of the obstructed segment were further assessed as well as the proximal and distal ends endoscopically and/or fluoroscopically; stent selection was based on these data and a goal of having at least $1.5 \mathrm{~cm}$ of stent on either side of the obstructed segment. A $0.035^{\prime \prime}$ straight-tip guidewire was passed through the obstructed segment under fluoroscopic guidance and at least another

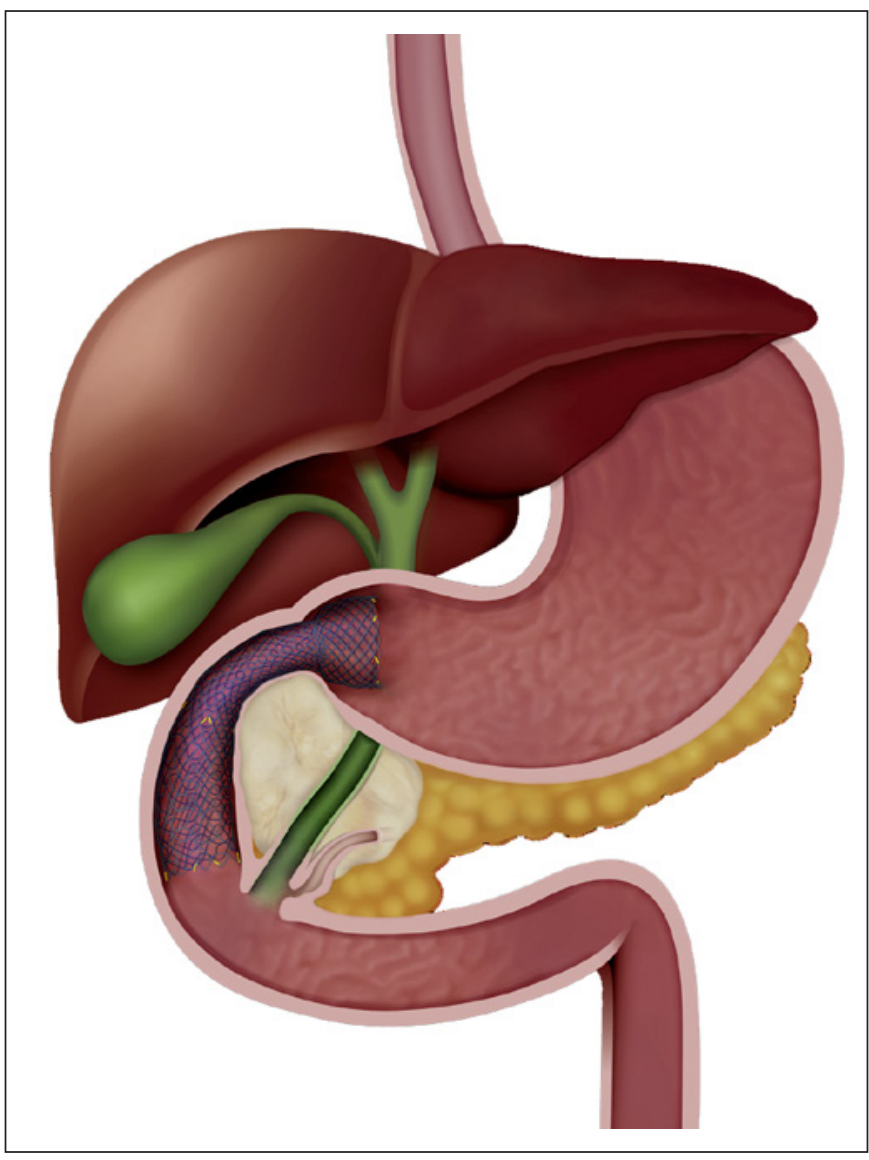

Fig. 1. Illustration of the duodenal HANAROSTENT ${ }^{\circledR}$. Shown here is a duodenal HANAROSTENT ${ }^{\circledR}$ placed for palliation of GDOO (in this case secondary to a pancreatic malignancy). A concerted effort is made to not obscure the major papilla in case of a future need for biliary stenting. The drawing illustrates (in gold) the radiopaque markers at the stent ends and in the center. GDOO, gastroduodenal outlet obstruction.

$10-15 \mathrm{~cm}$ distal to the obstruction for stability purposes. A duodenal HANAROSTENT ${ }^{\circledR}$ was advanced over the guidewire into the desired position and then deployed. The delivery catheter was withdrawn and location confirmed again endoscopically and fluoroscopically (Fig. 2). Patients were started on a clear liquid diet following the procedure, and diet was advanced as tolerated to a low-residue, mechanical soft diet.

\section{Data Collection and Analysis}

We retrospectively collected clinical (age, gender, etiology of GDOO, and length of follow-up), endoscopic, and radiologic data pertinent to patient and stent-related outcomes using a standardized data collection form. Technical success was defined as stent placement in the desired location during a single procedure without the need for adjustment. Clinical success was defined as resolution of vomiting, tolerating an oral diet (and discontinuation of parenteral nutrition, if applicable) following SEMS expansion, and not requiring further endoscopic or other procedures related to the 
SEMS (e.g., occlusion, migration, bleeding, or perforation) until last follow-up or death. Descriptive analyses were performed.

\section{Summary of Case Findings}

Clinical data for the 7 patients (5 men, 2 women, ages 44-61 years) meeting the study inclusion criteria are summarized in Table 1 . Three patients had primary gastroduodenal adenocarcinoma, and 4 patients had tumors of other origin (1 metastatic neuroendocrine tumor, 2 metastatic ovarian carcinoma, and 1 pancreatic adenocarcinoma). Five of the seven patients were receiving concomitant chemotherapy at the time of stent placement.

\section{Case Descriptions}

Cases of Primary Gastroduodenal Adenocarcinoma

Patient 1

A 44-year-old man with no significant past medical history presented with nausea, vomiting, and a 40-pound weight loss over the past 3 months. CT of the abdomen and pelvis revealed marked gastric distension with a narrowed and thickened pyloric channel. Endoscopic biopsy revealed poorly differentiated adenocarcinoma with signet-ring cell features. The patient subsequently underwent diagnostic laparoscopy and gastrojejunostomy. Two weeks later, the patient returned with abdominal pain and distention; repeat endoscopy revealed an edematous and ulcerated GJ anastomosis. A nasojejunal tube was placed, but this became repeatedly dislodged. Thus, the advanced endoscopy service was consulted, and a decision was made to place a $22 \times 90-$ mm duodenal HANAROSTENT ${ }^{\circledR}$ stent across the primary tumor/stricture in the pylorus to avoid trauma to the GJ anastomosis and allow more time for resolution of the anastomotic edema. However, within 2 days, the patient began to have obstructive symptoms again, and contrast-enhanced dynamic imaging demonstrated a closed entero-gastric loop. Thus, a nasojejunal tube was again placed, but this became dislodged within 4 days, similar to prior. Given the clinical circumstances, a $22 \times 120-\mathrm{mm}$ duodenal WallFlex ${ }^{\mathrm{TM}}$ stent (Boston Scientific, Natick, MA, USA) was placed across the gastrojejunal anastomosis into the efferent limb, after which the patient's symptoms improved markedly. Chemotherapy was started; on repeat imaging 12 months later, both stents remained patent and in good position, and the patient was continued on chemotherapy.

\section{Patient 2}

A 52-year-old man with a history of metastatic gastroduodenal adenocarcinoma on chemotherapy presented with new onset abdominal pain, nausea, and vomiting. CT of the abdomen and pelvis revealed progressive, circumferential thickening of the pylorus and proximal duodenum, as well as increased gastric distension. EGD was performed, revealing a 1-mm diameter crescentic opening thought to be the pyloric channel. Contrast duodenography using an inflated extraction balloon revealed an approximately 4-cm-long stricture spanning to the proximal second portion of the duodenum, concerning for progression of disease. A $22 \times 90$ mm duodenal HANAROSTENT ${ }^{\circledR}$ was deployed, with significant improvement in symptoms post-procedure. At 2-month followup, the patient remained on chemotherapy, was tolerating oral intake, and continued to be free from nausea and vomiting.

\section{Patient 3}

A 51-year-old man with metastatic signet-ring cell carcinoma of unknown primary origin presented with intermittent nausea

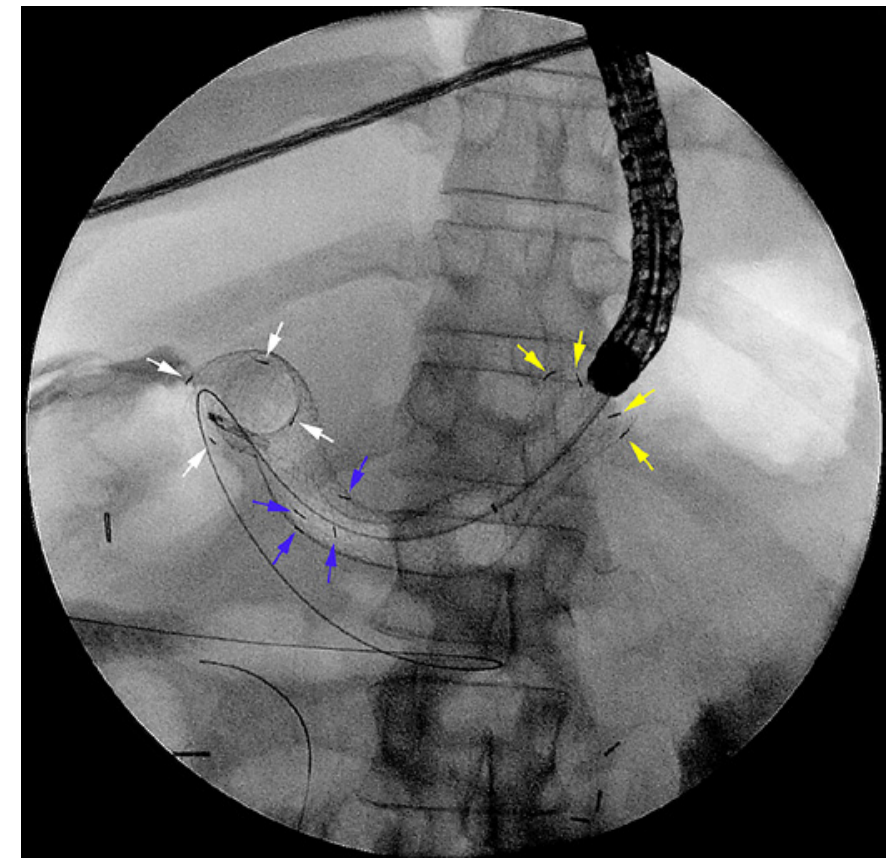

Fig. 2. Fluoroscopic view of the duodenal HANAROSTENT ${ }^{\circledR}$ immediately post-deployment across a long and tortuous segment of gastroduodenal obstruction. Twelve radiopaque markers are visible (4 proximal [yellow arrows], 4 middle [blue arrows], and 4 distal [white arrows]) and are useful in fluoroscopic stent visualization to facilitate precise endoscopic placement.

and vomiting. Serum laboratory tests were significant for an alkaline phosphatase of $1,280 \mathrm{U} / \mathrm{L}$, total bilirubin of $2.1 \mathrm{mg} / \mathrm{dL}$, AST of 379, and ALT of $507 \mathrm{U} / \mathrm{L}$. CT of the abdomen and pelvis revealed intrahepatic and extrahepatic biliary ductal dilation, a common bile duct diameter of $15 \mathrm{~mm}$, periportal lymphadenopathy, and a distended stomach with nonspecific thickening of the antrum and the proximal duodenum. Severe mucosal changes were seen beginning in the distal duodenal bulb, thus making duodenal origin of the tumor highly suspected. Fluoroscopy revealed a stricture at the level of the superior duodenal angle and stenosis of the majority of the second portion of the duodenum. Under endoscopic and fluoroscopic guidance, a $22 \times 90-\mathrm{mm}$ duodenal HANAROSTENT ${ }^{\circledR}$ was advanced through and deployed across the stricture, with the proximal end in the distal antrum and the distal end of the stent in the proximal third portion of the duodenum. A percutaneous biliary drain was subsequently placed by interventional radiology for the patient's biliary obstruction, and the patient was discharged with continuation of palliative chemotherapy. The patient died 1 month later from septic shock due to a suspected biliary tract infection.

\section{Cases of Other Primary Tumor Origin}

\section{Patient 4}

A 61-year-old man with no significant medical history presented with 8 months of intermittent nausea, vomiting, abdominal pain, and 30-pound weight loss. CT of the abdomen and pelvis 
Table 1. Summary of duodenal HANAROSTENT ${ }^{\circ}$ case features and outcomes

\begin{tabular}{|c|c|c|c|c|c|c|c|c|}
\hline Patient & $\begin{array}{l}\text { Age, } \\
\text { years }\end{array}$ & Gender & $\begin{array}{l}\text { Stent size, } \\
\mathrm{mm}\end{array}$ & Etiology of GDOO & $\begin{array}{l}\text { Technical } \\
\text { success }\end{array}$ & $\begin{array}{l}\text { Clinical } \\
\text { success }\end{array}$ & $\begin{array}{l}\text { Adverse } \\
\text { events }\end{array}$ & $\begin{array}{l}\text { Follow-up } \\
\text { time, } \\
\text { months }\end{array}$ \\
\hline 1 & 44 & $\mathrm{M}$ & $22 \times 90$ & $\begin{array}{l}\text { Gastric adenocarcinoma with signet-ring } \\
\text { cell features }\end{array}$ & Yes & Yes & No & 12.0 \\
\hline 2 & 52 & M & $22 \times 90$ & Duodenal carcinoma & Yes & Yes & No & 2.3 \\
\hline \multicolumn{9}{|c|}{ Other primary tumor origin } \\
\hline $4 \quad 1$ & 61 & $\mathrm{M}$ & $22 \times 90$ & Metastatic neuroendocrine tumor & Yes & Yes & No & $5.9^{\mathrm{a}}$ \\
\hline 5 & 48 & $\mathrm{~F}$ & $22 \times 120$ & Metastatic ovarian carcinoma & Yes & Yes & No & 5.1 \\
\hline 6 & 60 & $\mathrm{~F}$ & $22 \times 120$ & Metastatic ovarian carcinoma & Yes & Yes & No & $1.4^{\mathrm{a}}$ \\
\hline 7 & 56 & $\mathrm{M}$ & $22 \times 90$ & Unresectable pancreatic adenocarcinoma & Yes & Yes & No & 7.5 \\
\hline
\end{tabular}

GDOO, gastroduodenal outlet obstruction. ${ }^{\text {a }}$ Patient died at the end of the follow-up period.

revealed a distended stomach as well as numerous hypodense liver lesions and lymphadenopathy. Biopsy of one of the liver lesions revealed a grade 3 metastatic neuroendocrine tumor with Ki-67 index of approximately $50 \%$, of suspected gastric or pancreatic origin. On EGD, a large, ulcerated mass was found in the gastric body distorting the overall morphology of the mid- and distal stomach, as well as a short region of severe narrowing in the region of the pylorus. A $22 \times 90-\mathrm{mm}$ duodenal HANAROSTENT ${ }^{\circledR}$ was placed for palliation (Fig. 3). The patient was subsequently started on chemotherapy and radiation and discharged 10 days later. Approximately 6 months after discharge, the patient was readmitted after an episode of unwitnessed syncope thought to be due to a cardiac arrhythmia. Given no response to chemotherapy and a progressive decline in health, the patient was transitioned to comfort care and died several days later.

\section{Patient 5}

A 48-year-old woman with recurrent ovarian carcinoma and peritoneal carcinomatosis on salvage immunotherapy presented with intractable nausea and vomiting. There was no passage of oral contrast through the stomach on upper GI series. EGD revealed severe extrinsic compression of the mid-distal stomach distorting the overall anatomy and precluding visualization of the distal gastric body and antrum. A guidewire was navigated under endoscopic and fluoroscopic guidance through the obstructed segment and into the duodenum, and an extraction balloon was advanced over it. By serial contrast duodenography, the tortuous contour and distal extent of the obstructed segment were delineated and suggested feasibility of endoscopic stenting. Based on these data, a $22 \times 120$-mm duodenal HANAROSTENT ${ }^{\circledR}$ was deployed over the wire. Symptoms improved over the next week, and the patient was discharged home on a low-residue diet. The patient subsequently had repeated episodes of intermittent nausea and vomiting over the ensuing months, but the stent remained patent on imaging each time, and symptoms resolved with anti-emetic medication. She underwent radiation therapy and additional palliative chemo- therapy in an attempt to decrease tumor burden. Repeat CT 4 months later showed a patent gastric outlet stent, but also demonstrated dilated loops of small bowel with multiple transition points, areas of mesenteric and peritoneal soft tissue thickening, and implants tethering the bowel suggestive of multifocal mechanical small bowel obstruction. The patient was ultimately transitioned to hospice care.

\section{Patient 6}

A 60 -year-old woman with metastatic ovarian carcinoma presented with a large $140 \times 103 \times 127-\mathrm{mm}$ abdominal mass and inability to tolerate oral intake. An upper GI series with small bowel follow-through showed high-grade obstruction at the level of the third portion of the duodenum. On EGD, duodenal obstruction was noted at the inferior duodenal angle, consistent with extrinsic compression as suggested by CT. A $22 \times 120-\mathrm{mm}$ duodenal HANAROSTENT ${ }^{\circledR}$ was advanced over a guidewire and deployed across the obstructed segment. It was noted that the stent expanded to approximately only 6-7 $\mathrm{mm}$ at its narrowest point; nevertheless, the patient reported marked clinical improvement in nausea and vomiting over the next week and was restarted on chemotherapy. Given progression of the oncological disease, the patient was subsequently transitioned to hospice care and died 7 weeks after stent placement.

\section{Patient 7}

A 56-year-old man with disseminated tuberculosis and newly diagnosed unresectable stage III pancreatic adenocarcinoma status post-endoscopic biliary stenting presented with nausea, vomiting, and oral intolerance. An upper GI series showed no transit of contrast distal to the stomach. EGD revealed a moderate amount of solid and liquid food debris and stasis changes within the gastric body along with an angulated pyloric channel with extrinsic compression on and resultant stenosis of the mid-duodenal bulb. A 22 $\times 90-$ mm duodenal HANAROSTENT ${ }^{\circledR}$ was deployed across the region of obstruction, and the patient was discharged on post-pro-
Gastrointest Tumors 2021:8:1-7 DOI: $10.1159 / 000510350$
Fung et al. 

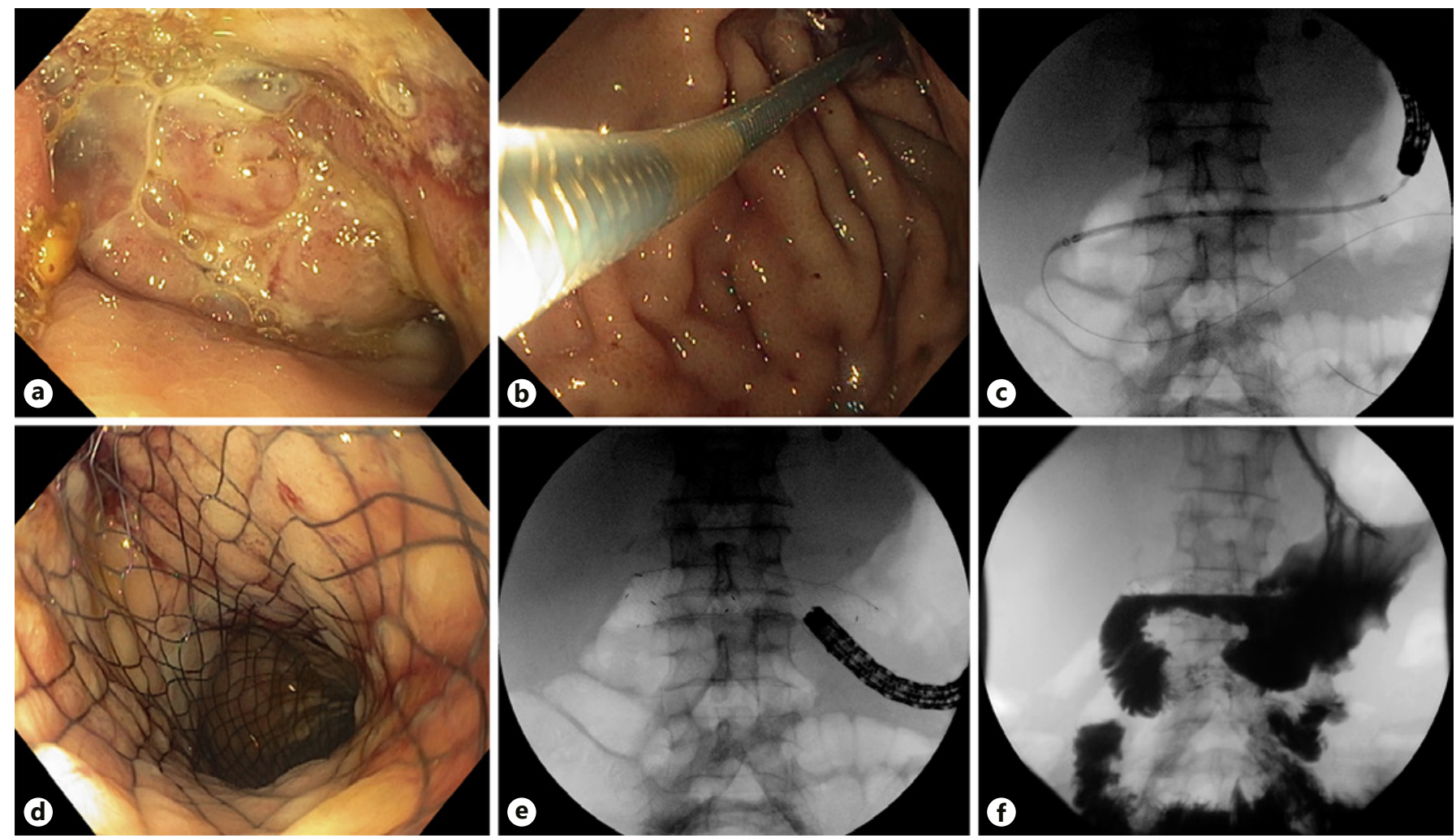

Fig. 3. Placement of the duodenal HANAROSTENT ${ }^{\circ}$ in a 61 -year-old male with a metastatic neuroendocrine tumor. (a) A large, ulcerated mass is found in the gastric body causing severe luminal narrowing. (b) A $22 \times 90$ $\mathrm{mm}$ duodenal HANAROSTENT ${ }^{\circ}$ is advanced over a guidewire through the region of greatest narrowing in the gastric antrum. c Appropriate positioning of the stent is confirmed fluoroscopically. d Deployed duodenal HANAROSTENT $^{\circ}$ as seen endoscopically and (e) fluoroscopically. $\mathbf{f}$ An upper GI series confirms stent patency.

cedure day 3 , tolerating a low-residue diet with no further nausea or vomiting. On most recent clinic follow-up (5 months poststenting), the patient was tolerating his oral diet and remained on chemotherapy.

\section{Discussion}

In this series, we found the use of the duodenal HANAROSTENT $^{\circledR}$, an uncovered SEMS, to be an effective method of palliation for malignant GDOO. The clinical and technical success rates with this specific duodenal stent were both $100 \%$, which is comparable, if not slightly higher, to what has been reported in the literature [5]. There were no stent-related adverse events peri-procedurally or during the mean follow-up time of 5 months (range 1-12 months). In several patients, stent placement was achieved in areas of severe stenosis and angulation (Fig. 4). One patient, despite having a patent stent, had ongoing nausea (believed to be due to peritoneal carcinomatosis and/or experimental chemotherapy) but was able to remain on an oral diet and off parenteral nutrition. In another patient with significant tumor burden, the stent showed persistent narrowing in its mid-portion, but the patient's obstructive symptoms improved, and an oral diet was tolerated without further vomiting. Three patients died during follow-up due to their underlying malignancy or complications thereof unrelated to the stent, and 4 remain alive.

The duodenal HANAROSTENT ${ }^{\circledR}$ is currently one of multiple SEMSs available for the palliation of GDOO. The WALLSTENT $^{\mathrm{TM}}$ (Boston Scientific) was the first stent approved by the US FDA for enteral use [7]. This uncovered SEMS is constructed of Elgiloy ${ }^{\circledR}$ (Elgiloy Inc., Elgin, IL, USA), an alloy of cobalt, chromium, nickel, iron, molybdenum, and manganese [8]. Although shown to be both effective and safe, at times this stent lacks adequate flexibility around angulations, and sharp distal ends may in- 


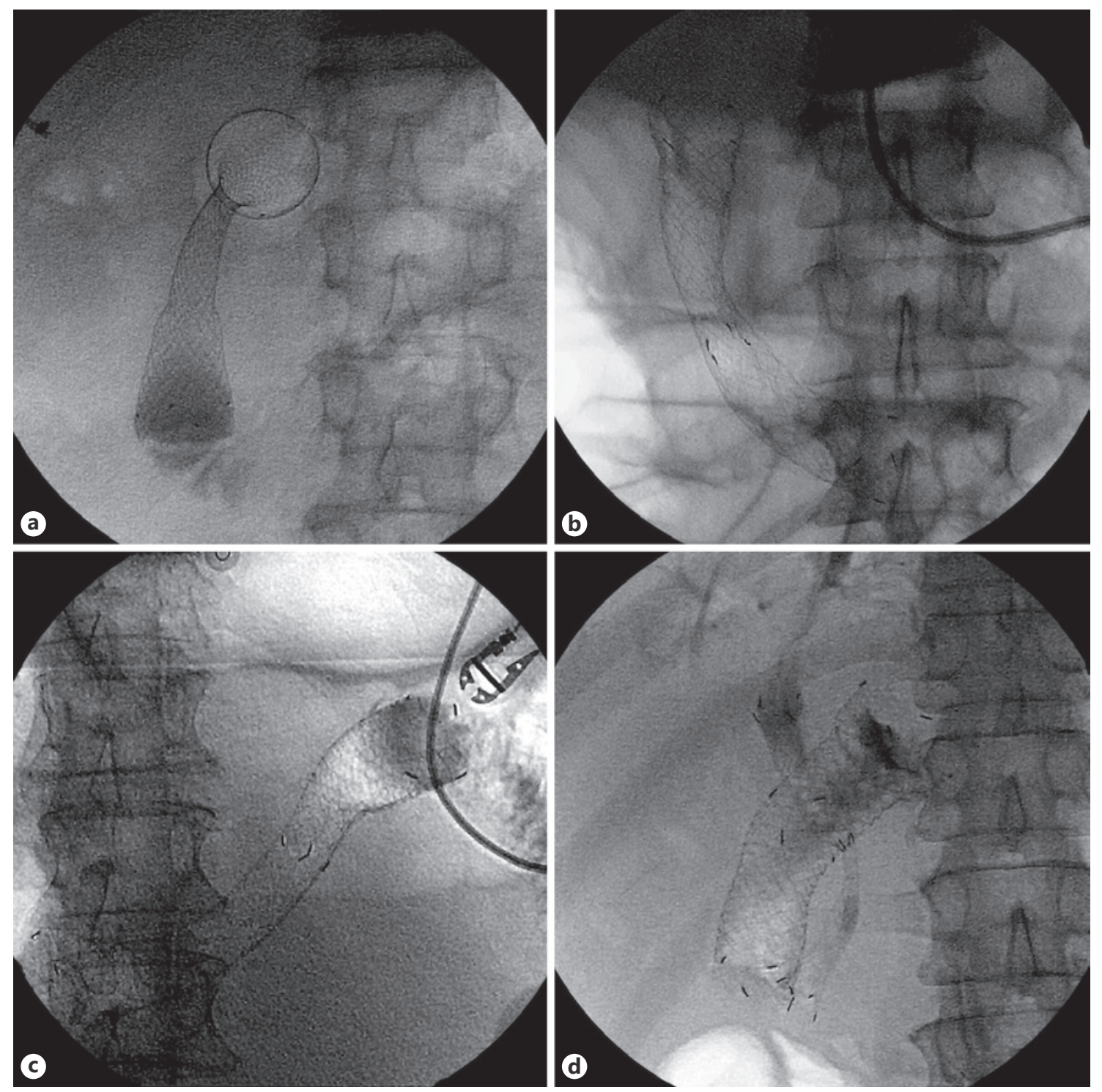

Fig. 4. Successful duodenal HANAROSTENT ${ }^{\circledR}$ placement as seen on fluoroscopic images obtained at the terminus of the index upper endoscopy. Patient $2(\mathbf{a})$, patient $3(\mathbf{b})$, patient $6(\mathbf{c})$, and patient $7(\mathbf{d})$.

crease the risk of perforation [9-11]. Today, numerous SEMS options exist, and the majority of SEMSs, including the HANAROSTENT ${ }^{\circledR}$, are constructed from nitinol, a nickel-titanium alloy that is soft and flexible yet has shape memory, thereby providing the stent a favorable balance between flexibility and maintaining luminal patency (i.e., radial expanding force) [3]; furthermore, its smoother wire ends may reduce the risk of bowel perforation [8]. These stent characteristics were supported by the findings in our cohort, with no recorded events of stent occlusion, migration, stent-related bleeding, or perforation.

\section{Conclusion}

The duodenal HANAROSTENT ${ }^{\circledR}$ is an alternative to other currently available SEMSs and may have comparative advantages. In this study, both technical and clinical success was achieved in all patients. As experience in the USA with this newly introduced SEMS grows, multicenter prospective data should be collected in order to better establish its relative safety and efficacy as well as potential cost savings. 


\section{Acknowledgement}

We would like to acknowledge Mr. Jeremy Bolt for technical details regarding the duodenal HANAROSTENT ${ }^{\circledR}$.

\section{Statement of Ethics}

The research was conducted ethically in accordance with the World Medical Association Declaration of Helsinki. All patients provided written informed consent for the care provided and for publishing photos and documentation of interventions performed.

\section{Conflict of Interest Statement}

The authors have no conflicts of interest to declare.

\section{Funding Sources}

The authors did not receive any funding.

\section{Author Contributions}

B.M.F. drafted the manuscript; B.E.K. and J.H.T. critically reviewed the manuscript; J.H.T. provided supervision; all authors provided critical input and approved of the manuscript.

\section{References}

1 Adler DG, Merwat SN. Endoscopic approaches for palliation of luminal gastrointestinal obstruction. Gastroenterol Clin North Am. 2006;35(1):65-82.

2 Upchurch E, Ragusa M, Cirocchi R. Stent placement versus surgical palliation for adults with malignant gastric outlet obstruction. Cochrane Database Syst Rev. 2018;5:CD012506.

3 Park J-S, Jeong S, Lee DH. Recent advances in gastrointestinal stent development. Clin Endosc. 2015;48(3):209-15.

4 LorenzoZúñiga V, Moreno-de-Vega V, Marín I, Boix J. Biodegradable stents in gastrointestinal endoscopy. World J Gastroenterol. 2014;20(9):2212-7.
5 van Halsema EE, Rauws EA, Fockens P, van Hooft JE. Self-expandable metal stents for malignant gastric outlet obstruction: a pooled analysis of prospective literature. World J Gastroenterol. 2015;21(43):12468-81.

6 Kato H, Tsutsumi K, Okada H. Recent advancements in stent therapy in patients with malignant gastroduodenal outlet obstruction. Ann Transl Med. 2017;5(8):186.

7 Lopera JE, Brazzini A, Gonzales A, Castaneda-Zuniga WR. Gastroduodenal stent placement: current status. Radiographics. 2004; 24(6):1561-73.
8 Boškoski I, Tringali A, Familiari P, Mutignani M, Costamagna G. Self-expandable metallic stents for malignant gastric outlet obstruction. Adv Ther. 2010;27:691-703.

9 Laasch H-U, Martin DF, Maetani I. Enteral stents in the gastric outlet and duodenum. Endoscopy. 2005;37(1):74-81.

10 Thumbe VK, Houghton AD, Smith MS. Duodenal perforation by a Wallstent. Endoscopy. 2000;32(6):495-7.

11 Telford JJ, Carr-Locke DL, Baron TH, Tringali A, Parsons WG, Gabbrielli A, et al. Palliation of patients with malignant gastric outlet obstruction with the enteral Wallstent: outcomes from a multicenter study. Gastrointest Endosc. 2004;60(6):916-20. 